\title{
PRODUCCIÓN DE XILITOL POR Candida guilliermondii A PARTIR DE FERMENTACIÓN DE RESIDUOS DE PALMA DE ACEITE
}

\section{XYLITOL PRODUCTION BY Candida guilliermondii FROM FERMENTATION OF WASTE OIL PALM}

\author{
Katherine Manjarres-Pinzón ${ }^{1}$, Mario Arias-Zabala ${ }^{2}$, Yuly Andrea Molina-Ramírez ${ }^{3}$, María Isabel Betancur-Nieto ${ }^{4}$ \\ Eduardo Rodríguez-Sandoval ${ }^{5}$
}

\begin{abstract}
${ }^{1}$ Ingeniera de Alimentos, Candidata Doctorado en Biotecnología, Facultad de Ciencias, Escuela de Química. Universidad Nacional de Colombia, calle 59a \# 63-20, Medellín, Antioquia, e-mail: jkmanjarresp@unal.edu.co; ${ }^{2}$ Ingeniero Químico, Ph.D, Profesor Titular, Facultad de Ciencias, Escuela de Química. Universidad Nacional de Colombia, calle 59a \# 63-20, Medellín, Antioquia, e-mail: marioari@unal.edu.co; ${ }^{3}$ Biotecnóloga, Facultad Ciencias de la Salud, Institución Universitaria Colegio Mayor de Antioquia, Carrera 78 \# 65-46 Medellín, Antioquia, e-mail: yuly.molina@colmayor.edu.co; ${ }^{4}$ Ingeniero Químico, MSc, Facultad de Ciencias Agrarias, Departamento de Ingeniería Agrícola y Alimentos, calle 59a \# 63-20. Universidad Nacional de Colombia, Medellín, Antioquia, e-mail: labcca med@unal.edu.co; ${ }^{5}$ Ingeniero Químico, Ph.D, Profesor Asociado, Facultad de Ciencias Agrarias, Departamento de Ingeniería Agrícola y Alimentos, calle 59a \# 63-20. Universidad Nacional de Colombia, Medellín, Antioquia, e-mail: edrodriguezs@unal.edu.co
\end{abstract}

Rev. U.D.C.A Act. \& Div. Cient. 19(2): 403-409, Julio-Diciembre, 2016

\section{RESUMEN}

La hidrólisis ácida diluida del residuo lignocelulósico de raquis de palma de aceite produce azúcares fermentables, como la xilosa, principal fuente de carbono para la producción de xilitol, por Candida guilliermondii. En este estudio, se evaluó el efecto de diferentes medios de cultivo y de condiciones de fermentación sobre la producción de xilitol, a partir de raquis de palma de aceite, utilizando $C$. guilliermondii. El hidrolizado ácido de raquis de palma suplementado con $4 \mathrm{~g} / \mathrm{L}$ extracto de levadura, $3 \mathrm{~g}\left(\mathrm{NH}_{4}\right)_{2} \mathrm{SO}_{4} / \mathrm{L}, 0,5 \mathrm{~g} / \mathrm{MgSO}_{4} .7 \mathrm{H}_{2} \mathrm{O} / \mathrm{L}$ y $0,1 \mathrm{gCaCl} 2.2 \mathrm{H}_{2} \mathrm{O} / \mathrm{L}$ mostró ser el mejor medio para el crecimiento de la levadura en cultivo sumergido, debido a que presentó los valores mayores, estadísticamente significativos, de la velocidad específica máxima de crecimiento, de $0,12 \mathrm{~h}^{-1}$ y producción de biomasa, de $5,5 \mathrm{~g} / \mathrm{L}$ $(p<0,05)$. Las condiciones de fermentación más apropiadas se obtuvieron con hidrolizado de raquis suplementado a $\mathrm{pH}$ de 5,5 , concentración inicial de xilosa de $17 \mathrm{~g} / \mathrm{L}$ y un inóculo de $3 \mathrm{~g} / \mathrm{L}$. La microaerobiosis mostró ser un factor importante en el proceso de fermentación, con un volumen de $40 \mathrm{~mL}$ de medio, en un matraz de $100 \mathrm{~mL}$, se produjo la mayor concentración de xilitol de 6,7 g/L ( $<<0,05)$.

Palabras clave: Residuos ligninocelulósicos, fermentación líquida, hidrólisis ácida, edulcorante, microaerobiosis.

\section{SUMMARY}

The dilute-acid hydrolysis of oil palm empty fruit bunch produces fermentable sugars such as xylose, main carbon source for xylitol production by Candida guilliermondii. The influence of different culture media and fermentation conditions were evaluated on the production of xylitol from oil palm empty fruit bunch using C. guilliermondii. The acid hydrolyzated oil palm empty fruit bunch supplemented with $4 \mathrm{~g}$ yeast extract/L, $3 \mathrm{~g}\left(\mathrm{NH}_{4}\right)_{2} \mathrm{SO}_{4} / \mathrm{L}, 0.5 \mathrm{~g} \mathrm{MgSO} 4.7 \mathrm{H}_{2} \mathrm{O} / \mathrm{L}$ y $0.1 \mathrm{~g} \mathrm{CaCl} 2.2 \mathrm{H}_{2} \mathrm{O} / \mathrm{L}$, was the best culture medium for the yeast growth in submerged culture because it had the highest values, statistically significant, of the specific growth rate $0.12 \mathrm{~h}^{-1}$ and biomass production $5.5 \mathrm{~g} / \mathrm{L}(\mathrm{p}<0,05)$. The suitable fermentation conditions were obtained with hydrolyzated oil palm empty fruit bunch supplemented at $\mathrm{pH}$ 5.5, initial xylose concentration of $17 \mathrm{~g} / \mathrm{L}$ and an inoculum of $3 \mathrm{~g} / \mathrm{L}$. Microaerobic condition is an important factor on the fermentation process, with a volume of $40 \mathrm{~mL}$ in a flask of $100 \mathrm{~mL}$, that produces the highest concentrations of xylitol $(6.7 \mathrm{~g} / \mathrm{L})(\mathrm{p}<0,05)$.

Key words: Lignocellulosic waste, liquid fermentation, acid hydrolysis, sweetener, microaerobic. 


\section{INTRODUCCIÓN}

La reutilización de residuos agroindustriales es un campo importante, a nivel biotecnológico, debido a su potencial, como materias primas, para la producción de energía y productos de valor agregado (Albuquerque et al. 2015); además, el uso de estos residuos contribuye a una reducción de la contaminación ambiental (Silva \& Roberto, 2001a). El proceso de beneficio del fruto de palma de aceite en Colombia es una de las agroindustrias con mayor generación de material contaminante. En términos conservadores, 1 ha de plantación de aceite de palma, anualmente, produce $55 \mathrm{t}$ de materia seca, en forma de biomasa fibrosa, mientras que la producción de aceite asciende a 5,5t. Adicionalmente, este cultivo tiene un alto rendimiento en la producción de aceite, si se compara con otras oleaginosas, como por ejemplo, con soya, 13 veces mayor; con girasol, 8 veces y con canola, 6,5 (Chang, 2014). Los principales residuos generados por esta agroindustria son: racimos vacíos, fibra, cuesco o cáscara del fruto y efluentes líquidos, principalmente. De los racimos de fruta fresca, alrededor de 20 a $23 \%$, son racimos vacíos o raquis; 11 a $14 \%$ son fibras; 5 a $7 \%$ es cuesco o cáscara del fruto y 65 a $85 \%$ son efluentes líquidos (García et al. 2010). La producción nacional de racimos de fruta fresca, para el 2014, fue de 5,4 millones de toneladas, lo cual, representa un estimativo de 1,24 millones de racimos vacíos o raquis de palma (Fedepalma, 2015). Los racimos vacíos están conformados por celulosa (23,7-65\%), hemicelulosa (20,58$33,52 \%)$, lignina $(14,1-30,4 \%)$ y cenizas $(1,3-13 \%)$ (Chang, 2014). El polisacárido más abundante de la hemicelulosa es el xilano, que puede ascender a 95\% del total de los polisacáridos no celulósicos de la biomasa. El porcentaje de xilano en los raquis de palma varía entre 20-27\% (Shatalov \& Pereira, 2012; Chin et al. 2015). Estos residuos, por su composición, constituyen un sustrato potencial para obtener azúcares fermentables, tales como glucosa y xilosa, que pueden ser convertidos, biotecnológicamente, en productos de valor económico, como xilitol y etanol (Pereira et al. 2011; Albuquerque et al. 2015).

El pretratamiento de lignocelulosa, para obtener azúcares fermentables, es un paso esencial para la conversión con fermentación microbiana. Una variedad de pretratamientos, como el mecánico, químico y biológico, han sido desarrollados para cambiar la estructura y la composición química de la lignocelulosa y así mejorar la producción de azúcares (Duangwang \& Sangwichien, 2015; Carvalho et al. 2003). Entre estos métodos, el pretratamiento con hidrolisis ácida puede ser aplicado con ácido diluido o concentrado, aunque el empleo de ácidos concentrados es menos deseable, porque se forman compuestos inhibitorios. La hidrolisis ácida diluida es el método más aplicado generalmente y el más estudiado entre los pretratamientos químicos. El objetivo principal de la hidrolisis ácida es solubilizar la fracción de hemicelulosa de la biomasa e incrementar la accesibilidad de la celulosa por parte de las enzimas (Zhang et al. 2012).

Algunas especies de levaduras, se encuentran entre los mejores productores de xilitol, fermentando la xilosa, mediante la enzima xilosa-reductasa (XR), dependiente de la Nicotinamida Adenina Dinucleótido Fosfato $\left(\mathrm{NADPH}^{+}\right)$, produciendo xilitol (Tomotani et al. 2009; Arruda et al. 2011). Entre las levaduras más destacadas en este proceso, se registran Candida guilliermondii (Acosta et al. 2005; Arruda et al. 2011; Sene et al. 2011), Candida tropicalis (Ling et al. 2011) y Pichia guilliermondii (Zou et al. 2010).

El xilitol, un alcohol pentahidroxilado de la xilosa, puede ser producido química o bioquímicamente, a partir de xilosa (Acosta et al. 2005; Arruda et al. 2011). Este poliol es usado como edulcorante en la industria de alimentos, así como en productos para la higiene bucal, farmacéuticos y cosméticos. Tiene propiedades anticariogénicas y se puede usar por personas diabéticas, ya que su metabolismo es independiente de la insulina; además, es un compuesto benéfico para personas obesas, porque contribuye a la baja formación de grasa en el tejido adiposo (Arruda et al. 2011; Sene et al. 2011).

Diferentes estudios enfocados en la producción de xilitol, vía fermentación, han evaluado diversas condiciones de fermentación, como la aireación, a fin de aumentar su rendimiento (Soleimani \& Tabil, 2014), el pH (Cheng et al. 2009), la concentración de sustrato (Chang, 2014; Camargo et al. 2015), el tiempo y sistemas de fermentación (Carvalho et al. 2003). También, se han evaluado las condiciones de hidrólisis (Pereira et al. 2011; Sene et al. 2011), la adaptación de la levadura y los métodos de detoxificación (Kamal et al. 2011). El objetivo de este estudio fue evaluar diferentes medios de cultivo y diversas condiciones de fermentación, en la producción de xilitol por Candida guilliermondii, a partir de hidrolizados ácidos de raquis de palma de aceite, a escala de matraz agitado.

\section{MATERIALES Y MÉTODOS}

Materiales. Los racimos vacíos de frutos o raquis de palma fueron suministrados por la planta de beneficio Palmares del Oriente S.A.S. Los racimos se lavaron, secaron y molieron, en una máquina pica pasto (Molino \#1, JM. ESTRADA.S.A, La Estrella, Colombia), hasta un tamaño de partícula de 3 a $5 \mathrm{~mm}$.

Hidrólisis ácida del raquis de palma. La hidrólisis ácida de los raquis, se realizó en matraces Erlenmeyer de $500 \mathrm{~mL}$, con una relación 1:8 sólido/líquido, tomando $40 \mathrm{~g}$ del raquis y $320 \mathrm{~mL}$ de ácido sulfúrico, diluido al $2 \%$. Los matraces fueron esterilizados por $30 \mathrm{~min}$, a $121^{\circ} \mathrm{C}$ y posteriormente 
sumergidos en un baño de hielo, para detener la hidrólisis. Finalmente, se filtró todo el contenido del hidrolizado y se ajustó el pH a 5,0, 5,5 y 6,0 con $\mathrm{NaOH}$ al 98\%, para los diferentes tratamientos.

Crecimiento de la levadura en diferentes medios. El crecimiento de Candida guilliermondiii ATCC 6260, se determinó en los siguientes medios, a $30^{\circ} \mathrm{C}$ y 108 rpm: a) medio líquido de glucosa, peptona y extracto de levadura (YPG); b) hidrolizado de raquis de palma (HR) y, c) hidrolizado de raquis de palma suplementado (HRS), con $4 \mathrm{~g}$ extracto de levadura/L, $3 \mathrm{~g}\left(\mathrm{NH}_{4}\right)_{2} \mathrm{SO}_{4} / \mathrm{L}, 0,5 \mathrm{~g} \mathrm{MgSO}_{4} .7 \mathrm{H}_{2} \mathrm{O} / \mathrm{L}$ y $0,1 \mathrm{~g}$ $\mathrm{CaCl}_{2} .2 \mathrm{H}_{2} \mathrm{O} / \mathrm{L}$. La biomasa, se determinó por peso seco. En el método de peso seco, se tomaron $10 \mathrm{~mL}$ de muestra, se centrifugó a 3900rpm, durante $20 \mathrm{~min}$, a $25^{\circ} \mathrm{C}$, se lavó dos veces con agua destilada y se secó en cajas Petri, a $60^{\circ} \mathrm{C}$, por $72 \mathrm{~h}$, para luego registrar el peso seco (Niño-Camacho \& Torres-Sáenz, 2010). De las curvas de crecimiento, se obtuvieron los siguientes parámetros: tiempo de fase exponencial, tiempo de duplicación (h) y velocidad específica máxima de crecimiento $\left(\mathrm{h}^{-1}\right)$.

Fermentación a diferentes condiciones. Se seleccionó el mejor medio de cultivo empleado, con base en el menor tiempo de duplicación de la biomasa. Las condiciones de fermentación evaluadas fueron: $\mathrm{pH}(5,0$ y 5,5), concentración de inóculo ( 3 y $5 \mathrm{~g} / \mathrm{L}$ ) y concentración de xilosa (17 y 37g/L). La fermentación, se llevó a cabo en matraces Erlenmeyer de $100 \mathrm{~mL}$, tomando $47 \mathrm{~mL}$ de medio de cultivo, se incubó en un agitador, a 200rpm y $30^{\circ} \mathrm{C}$, por 96 h. Los parámetros evaluados fueron: concentración de xilitol, rendimiento de producto con respecto al sustrato (Yp/s) y velocidad volumétrica de formación de producto (Qp). Las concentraciones de xilosa y de xilitol fueron determinadas por HPLC (Shimadzu Prominence 20A, Kyoto, Japan), con una columna Aminex HPX-87H (Biorad) y un detector RI (Piñeros-Castro et al. 2011).

Microaerobiosis. Se estudiaron diferentes condiciones de aireación a escala de matraz Erlenmeyer de $100 \mathrm{~mL}$, con variaciones en el volumen de medio efectivo, utilizado de 40, 60, 80 y 90mL (Villalba et al. 2009). La microaerobiosis, se determinó utilizando el medio de cultivo previamente seleccionado y un medio mínimo de xilosa (MMX), los cuales, fueron inoculados con $5 \mathrm{~g}$ de la levadura/L, a $200 \mathrm{rpm}$ y $30^{\circ} \mathrm{C}$, con un pH y una concentración inicial de xilosa específicos. Se realizaron al menos dos repeticiones de cada tratamiento.

Diseño experimental. En las pruebas de crecimiento de la levadura y microaerobiosis, se empleó un diseño factorial de una sola vía, tomando como factores el tipo de medio y el volumen efectivo, respectivamente. Además, en las pruebas de fermentación, se aplicó un diseño factorial $2^{k}$ completamente aleatorizado, con los siguientes factores:
pH, concentración de inóculo y concentración de xilosa. Los datos experimentales, se sometieron a ANOVA, con un nivel de significancia del $5 \%$ y se utilizó diferencia mínima significativa (LSD), para comparar los tratamientos con diferencias significativas. El análisis estadístico, se aplicó mediante Statgraphics plus 5,1. Los resultados se presentan como el promedio \pm desviación estándar.

\section{RESULTADOS Y DISCUSIÓN}

Cinéticas de crecimiento en diferentes medios. Las curvas de crecimiento de $C$. guilliermondii en los medios YPG, HR y HRS, se observan en la figura 1 . En la tabla 1 , se reportan los valores de la velocidad específica máxima de crecimiento, el tiempo de duplicación y el tiempo de la fase exponencial. El tratamiento que se seleccionó para el montaje de las fermentaciones en matraz agitado fue HRS, debido a que presentó el mayor valor de la velocidad específica máxima de crecimiento $\left(0,12 \mathrm{~h}^{-1}\right)$, correspondiente a un tiempo de duplicación de $5,77 \mathrm{~h}$ y la mayor concentración final de biomasas $(5,5 \mathrm{~g} / \mathrm{L})$. Los compuestos usados para suplementar el hidrolizado excluyen cualquier limitación del crecimiento de la levadura, debido a una deficiencia nutricional. Adicionalmente, en la curva de crecimiento con YPG, se observó una fase de adaptación prolongada de 20h, dado que la cepa se mantuvo por debajo de su temperatura óptima $\left(30^{\circ} \mathrm{C}\right)$. El comportamiento de la levadura en HR no muestra una disminución de la concentración de biomasa en las primeras horas de la fermentación, a pesar de la presencia de compuestos tóxicos, como por ejemplo, hidroximetilfurfural $(0,0023 \mathrm{~g} / \mathrm{L})$, determinado en ensayos preliminares. Este compuesto es conocido como inhibidor en el crecimiento de la levadura; sin embargo, la concentración obtenida en este estudio no fue lo suficientemente alta para afectar metabólicamente la levadura (Carvalheiro et al. 2005).

Fermentación a escala de matraz. En la tabla 2, se observan los resultados de Yp/s, Qp y concentración final de xilosa, para las diferentes condiciones de fermentación. El tratamiento que mostró la mayor producción de xilitol $(2,025 \mathrm{~g} / \mathrm{L})$ corresponde a un $\mathrm{pH}$ de 5,5, concentración inicial de xilosa de $17 \mathrm{~g} / \mathrm{L}$ y un inóculo de $3 \mathrm{~g} / \mathrm{L}$. A esas mismas condiciones, Yp/s y Qp fueron de 0,094 g/g y 0,021 $\mathrm{g} / \mathrm{L} . \mathrm{h}$, respectivamente. Otros estudios sobre fermentación con hidrolizados de residuos agroindustriales, utilizando C. guilliermondii para la producción de xilitol, reportan rendimientos $\mathrm{de} 0,7 \mathrm{~g}$ de xilitol $/ \mathrm{g}$ de sustrato bajo, condiciones semiaeróbicas a partir de $150 \mathrm{~g}$ xilosa/L inicial (Mohamad et al. 2014). Así, por ejemplo, Arruda et al. (2011), al utilizar C. guilliermondii para la producción de xilitol con bagazo de caña, halló que la máxima producción fue de $50,5 \mathrm{~g} / \mathrm{L}$ a escala matraz $(125 \mathrm{~mL})$, con un rendimiento $\left(\mathrm{Y}_{\mathrm{p} / \mathrm{s}}\right)$ de $0,81 \mathrm{~g} / \mathrm{g}$ y una productividad volumétrica $\left(Q_{p}\right)$ de 0,60g/L.h, después 


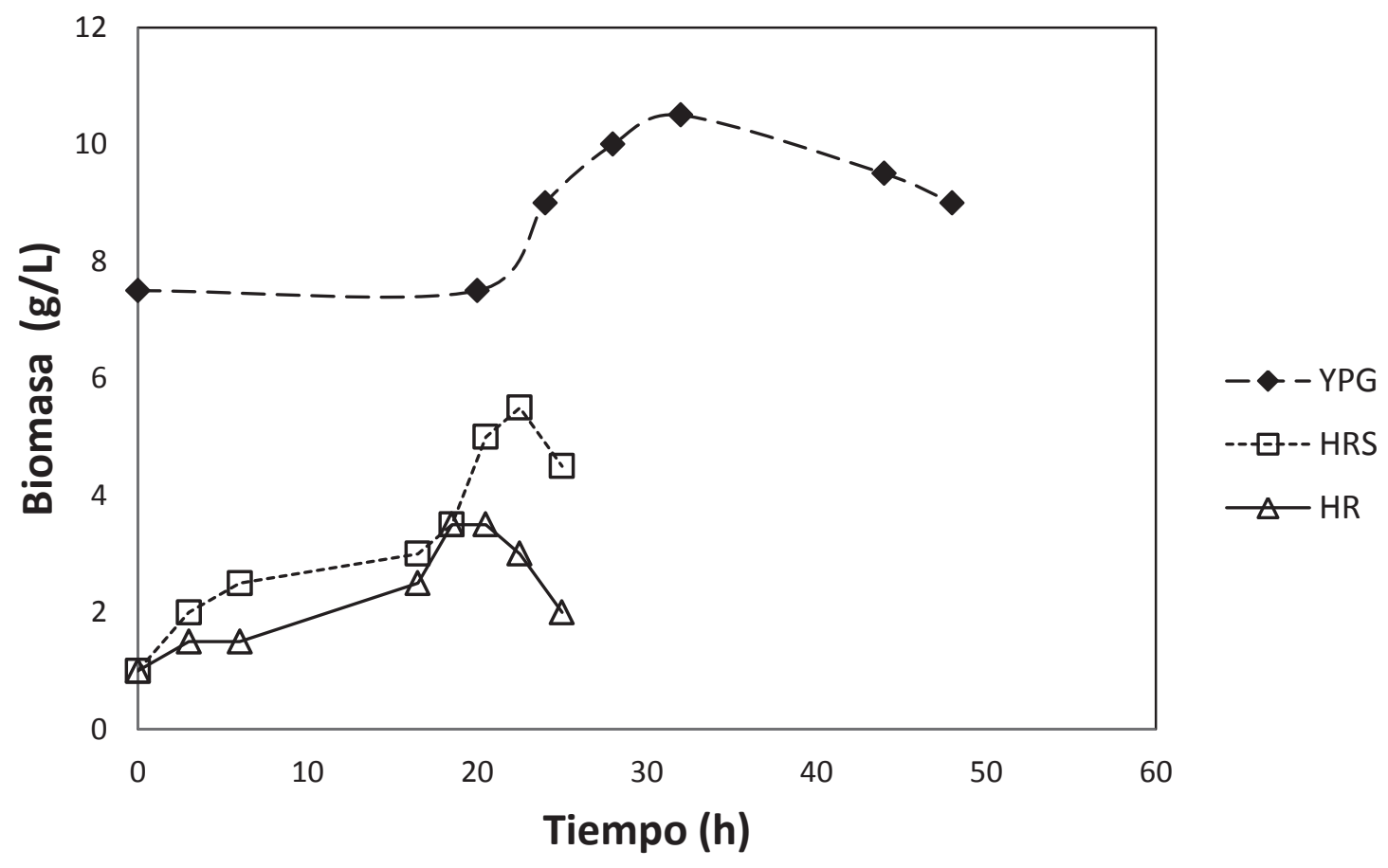

Figura 1. Cinética de crecimiento de Candida guilliermondii en medios YPG, HR y HRS, a $30^{\circ} \mathrm{C}$ y $108 \mathrm{rpm}$.

Tabla 1. Parámetros de las cinéticas de crecimiento de Candida guilliermondii en diferentes medios de cultivo.

\begin{tabular}{|c|c|c|c|}
\hline Medio de cultivo & $\begin{array}{c}\text { Tiempo fase } \\
\text { exponencial (h) }\end{array}$ & $\begin{array}{c}\text { Velocidad específica } \\
\text { máxima de crecimiento } \\
\mathbf{( h}^{-1} \mathbf{)}\end{array}$ & $\begin{array}{c}\text { Tiempo de } \\
\text { duplicación (h) }\end{array}$ \\
\hline YPG & 8 & 0,03 & 23,10 \\
\hline HR & 12,5 & 0,06 & 11,55 \\
\hline HRS & 4 & 0,12 & 5,77 \\
\hline
\end{tabular}

YPG: medio líquido de glucosa, peptona y extracto de levadura; HR: hidrolizado de raquis de palma; HRS: hidrolizado de raquis de palma suplementado.

de 96 h de fermentación, a un pH 5,5 y $30^{\circ} \mathrm{C}$. También, se ha reportado una producción de xilitol de $52 \mathrm{~g} / \mathrm{L}$, con un factor de rendimiento de $0,65 \mathrm{~g} / \mathrm{g}$, usando C. guilliermondii e hidrolizado de cascarilla de arroz, como sustrato (Silva \& Roberto, 2001b). Una posible causa del bajo rendimiento de xilitol en este estudio podría ser la baja concentración de xilosa inicial, de aproximadamente $17-37 \mathrm{~g} / \mathrm{L}$. Una mayor concentración de sustrato aumenta la concentración final de producto en el hidrolizado de raquis (Silva \& Mussatto, 2006).
Microaerobiosis. La disponibilidad de oxígeno es un factor importante en la producción biotecnológica de xilitol, a partir de D-xilosa. En condiciones limitadas de oxígeno, la fosforilación oxidativa no puede volver a oxidar todo el Dinucleótido de Nicotinamida y Adenina (NADH) generado, lo cual, conduce a una acumulación de xilitol, a nivel intracelular (Albuquerque et al. 2014). Además, la presencia de alta cantidad de oxígeno favorece la conversión de xilosa a biomasa, mientras que una baja cantidad de oxígeno aumenta el rendimiento de xilitol (Aguiar et al. 2002; Carvalho et al. 2003). La producción de xilitol por medio 
de microaerobiosis, a un $\mathrm{pH}$ de 5,5 y una concentración inicial de xilosa de $17 \mathrm{~g} / \mathrm{L}$, en este estudio, se observa en la tabla 3. Los medios de cultivo estudiados fueron HRS y MMX. El mejor resultado, se obtuvo con HRS a $40 \mathrm{~mL}$ de medio, en matraces de $100 \mathrm{~mL}$, con una producción de $6,7 \mathrm{~g}$ xilitol/L. Esto indica, que la microaerobiosis a un volumen de $40 \mathrm{~mL}$, en un matraz de $100 \mathrm{~mL}$ y en cultivo HRS, es apropiado para la producción de xilitol, tomando en cuenta los valores reportados de los demás tratamientos (Tabla 3 ); sin embargo, son valores bajos comparados con los de otros estudios (Silva \& Roberto, 2001b; Arruda et al. 2011). Es necesario tener en cuenta que estos estudios emplean mayor cantidad de sustrato, lo que indica la aplicación de un proceso de concentración de la xilosa, procedimiento que no fue necesario para obtener xilitol a partir de hidrolizado de raquis de palma. La microaerobiosis para la producción de xilitol es importante, debido a que la acumulación de xilitol en la levadura está directamente asociada al desequilibrio redox, limitando las coenzimas, para mayor generación de producto (Mohamad et al. 2014). Previamente, se ha reportado que el volumen de medio ideal es de $40 \%$, para el crecimiento de biomasa y $88 \%$, para la producción de xilitol en microaerobiosis, bajo condiciones controladas de $\mathrm{O}_{2}$, inóculo y pH, entre otros (Aguiar et al. 2002); sin embargo, Nolleau et al. (1993) reportaron un rendimiento $\left(\mathrm{Y}_{\mathrm{p} / \mathrm{s}}\right)$ en la producción de xilitol, de $0,69 \mathrm{~g} / \mathrm{g}$, con C. guilliermondii, bajo condiciones de aerobiosis, con una concentración inicial de $300 \mathrm{~g}$ xilosa/L. Dicha condición de oxígeno disponible presentó una alta densidad celular, garantizando óptima generación de xilitol.

En general, el crecimiento de la levadura C. guilliermondii ATCC 6260 en hidrolizados de raquis de palma suplementado

Tabla 2. Fermentación en matraz por 96h, con diferentes concentraciones de inóculo, sustrato y pH.

\begin{tabular}{|c|c|c|c|c|c|}
\hline \multicolumn{3}{|c|}{ Condiciones de fermentación evaluadas } & \multirow{2}{*}{ Xilitol (g/L) } & \multirow{2}{*}{$\mathrm{Yp} / \mathrm{s}(\mathrm{g} / \mathrm{g})$} & \multirow{2}{*}{ Qp (g/L.h) } \\
\hline $\mathrm{pH}$ & Xilosa inicial $(\mathrm{g} / \mathrm{L})$ & Inóculo (g/L) & & & \\
\hline \multirow{4}{*}{5,5} & 17 & 3 & $2,025 \pm 0,03^{d}$ & 0,094 & 0,021 \\
\hline & 17 & 5 & $1,426 \pm 0,02^{c}$ & 0,076 & 0,018 \\
\hline & 37 & 3 & $1,317 \pm 0,00^{c}$ & 0,058 & 0,014 \\
\hline & 37 & 5 & $1,141 \pm 0,02^{c}$ & 0,046 & 0,012 \\
\hline \multirow{4}{*}{5} & 17 & 3 & $0,700 \pm 0,01^{a}$ & 0,089 & 0,007 \\
\hline & 17 & 5 & $0,701 \pm 0,02^{a}$ & 0,071 & 0,007 \\
\hline & 37 & 3 & $0,683 \pm 0,08^{a}$ & 0,040 & 0,007 \\
\hline & 37 & 5 & $0,799 \pm 0,07^{b}$ & 0,033 & 0,008 \\
\hline
\end{tabular}

*Las diferencias de letras en la misma columna indican una diferencia significativa $(p<0.05)$, de acuerdo a la prueba LSD.

Tabla 3. Microaerobiosis con hidrolizado suplementado (HRS), medio mínimo de xilosa (MMX) y cepa nativa de Candida guilliermondii.

\begin{tabular}{|c|c|c|}
\hline \multirow{2}{*}{$\begin{array}{c}\text { Volumen } \\
\text { efectivo }(\mathrm{mL})\end{array}$} & \multicolumn{2}{|c|}{ Medio } \\
\cline { 2 - 3 } & HRS & MMX \\
\cline { 2 - 3 } & Xilitol $(\mathbf{g} / \mathbf{L})^{\text {Xilitol }(\mathbf{g} / \mathbf{L})}$ \\
\hline 40 & $6,745 \pm 0,30^{\mathrm{c}}$ & $0,323 \pm 0,03^{\mathrm{b}}$ \\
\hline 60 & $0,143 \pm 0,01^{\mathrm{a}}$ & $0,037 \pm 0,01^{\mathrm{a}}$ \\
\hline 80 & $0,317 \pm 0,01^{\mathrm{b}}$ & $0,035 \pm 0,00^{\mathrm{a}}$ \\
\hline 90 & $0,367 \pm 0,02^{\mathrm{b}}$ & $0,046 \pm 0,01^{\mathrm{a}}$ \\
\hline
\end{tabular}

*Las diferencias de letras en la misma columna indican una diferencia significativa $(\mathrm{p}<0,05)$, de acuerdo a la prueba LSD. 
(HRS) presentó los mayores valores de velocidad específica máxima de crecimiento $\left(0,12 \mathrm{~h}^{-1}\right)$, correspondiente a un tiempo de duplicación de $5,77 \mathrm{~h}$ y de producción de biomasa de 5,5g/L. Las condiciones de fermentación con cultivo HRS que tuvieron la mayor producción de xilitol fueron $\mathrm{pH}$ de 5,5 , concentración inicial de xilosa de $17 \mathrm{~g} / \mathrm{L}$ y un inóculo de $3 \mathrm{~g} / \mathrm{L}$. Además, al aplicar microaerobiosis, con un volumen de $40 \mathrm{~mL}$, en matraces de $100 \mathrm{~mL}$, a las condiciones de fermentación anteriormente mencionadas, aumenta la concentración de xilitol a $6,7 \mathrm{~g} / \mathrm{L}$, lo que representa un parámetro de gran importancia para aumentar la producción de xilitol, utilizando esta cepa

Agradecimientos: Los autores agradecen a Colciencias, por la beca otorgada a Katherine Manjarres-Pinzón. Conflictos de interés: El manuscrito fue preparado y revisado con la participación de todos los autores, quienes declaramos que no existe conflicto de interés que ponga en riesgo la validez de los resultados presentados. Financiación: Este estudio fue financiado por la Universidad Nacional de Colombia, sede Medellín.

\section{BIBLIOGRAFÍA}

1. ACOSTA, E.; ALMEIDA, J.B.; GIULIETTI, M.; FRADE, J.A.; NÁPOLES, A.L. 2005. Producción de xilitol en fermentador de 15 litros. ICIDCA. 29(3):45-51.

2. AGUIAR, W.B.; FARIA, L.F.F.; COUTO, M.A.P.G.; ARAUJO, O.Q.F.; PEREIRA, N. 2002. Growth model and prediction of oxygen transfer rate for xylitol production from D-xylose by Candida guilliermondii. Biochem. Eng. J. 12:49-59.

3. ALBUQUERQUE, T.L.; GOMES, S.D.L.; MARQUES, J.E.; SILVA, I.J.; ROCHA, M.V.P. 2015. Xylitol production from cashew apple bagasse by Kluyveromyces marxianus CCA510. Catal. Today 255:33-40.

4. ALBUQUERQUE, T.L.; SILVA, I.J.; MACEDO, G.R.; D, VALDEREZ, M.; ROCHA, M.V.P. 2014. Biotechnological production of xylitol from lignocellulosic wastes: A review. Process Biochem. 49:1779-1789.

5. ARRUDA, P.V.; RODRIGUES, R.C.L.B.; SILVA, D.D.V.; FELIPE, M.G.A. 2011. Evaluation of hexose and pentose in pre-cultivation of Candida guilliermondii on the key enzymes for xylitol production in sugarcane hemicellulosic hydrolysate. Biodegradation. 22:81522.

6. CAMARGO, D.; SENE, L.; VARIZ, D.I.L.S.; FELIPE, M.G.A. 2015. Xylitol bioproduction in hemicellulosic hydrolysate obtained from sorghum forage biomass. Appl. Biochem. Biotechnol. 175(8):3628-3642.

7. CARVALHEIRO, F.; DUARTE, L.C.; LOPES, S.; PARAJÓ, J.C.; PEREIRA, H.; GIRIO, F.M. 2005. Evaluation of the detoxification of brewery's spent grain hydrolysate for xylitol production by Debaryomyces hansenii CCMI 941. Process Biochem. 40:1215-1223.

8. CARVALHO, W.; SILVA, S.S.; SANTOS, J.C.; CONVERTI, A. 2003. Xylitol production by Ca-alginate entrapped cells: comparison of different fermentation systems. Enzyme Microb. Technol. 32:553-559.

9. CHANG, S.H. 2014. An overview of empty fruit bunch from oil palm as feedstock for bio-oil production. Biomass Bioenerg. 62:174-181.

10. CHENG, K.K.; ZHANG, J.A.; LING, H.Z.; PING, W.X.; HUANG, W.; GE, J.P.; XU, J.M. 2009. Optimization of $\mathrm{pH}$ and acetic acid concentration for bioconversion of hemicellulose from corncobs to xylitol by Candida tropicalis. Biochem. Eng. J. 43:203-207.

11. CHIN, S.X.; CHIA, C.H.; ZAKARIA, S.; FANG, Z.; AHMAD, S. 2015. Ball milling pretreatment and diluted acid hydrolysis of oil palm empty fruit bunch (EFB) fibres for the production of levulinic acid. J. Taiwan Inst. Chem. Eng. 52:85-92.

12. DUANGWANG, S.; SANGWICHIEN, CH. 2015. Utilization of Oil Palm Empty Fruit Bunch Hydrolysate for Ethanol Production by Baker's Yeast and Loog-Pang. Energy Procedia. 79:157-162.

13. FEDEPALMA. 2015. Minianuario Estadístico 2015. Federación Nacional de Cultivadores de Palma de Aceite. Bogota. 64p.

14. GARCÍA, J.A.; CÁRDENAS, M.M.; YÁÑEZ, E.E. 2010. Generación y uso de biomasa en plantas de beneficio de palma de aceite en Colombia. Palmas. 31(2):4148

15. KAMAL, S.M.M.; MOHAMAD, N.L.; ABDULLAH, A.G.L.; ABDULLAH, N. 2011. Detoxification of sago trunk hydrolysate using activated charcoal for xylitol production. Procedia Food Sci. 1:908-913.

16. LING, H.; CHENG, K.; GE, J.; PING, W. 2011. Statistical optimization of xylitol production from corncob hemicellulose hydrolysate by Candida tropicalis HDY-02. N. Biotechnol. 28:673-678. 
17. MOHAMAD, N.L.; KAMAL, S.M.M.; MOKHTAR, M.N. 2014. Xylitol biological production: A review of recent studies. Food Rev. Int. 31(1):74-89.

18. NIÑO-CAMACHO, L.; TORRES-SÁENZ, R. 2010. Implementación de diferentes técnicas analíticas para la determinación de biomasa bacteriana de cepas Pseudomonas putida biodegradadoras de fenol. Rev. Ion. 23:41-46.

19. NOLLEAU, V.; DELGENES, J.P.; NAVARRO, J.M. 1993. Xylitol production from xylose by two yeast strains: Sugar tolerance. Curr. Microbiol. 27:191-197.

20. PEREIRA, R.S.; MUSSATTO, S.I.; ROBERTO, I.C. 2011. Inhibitory action of toxic compounds present in lignocellulosic hydrolysates on xylose to xylitol bioconversion by Candida guilliermondii. J. Ind. Microbiol. Biotechnol. 38:71-78.

21. PIÑEROS-CASTRO, Y.; VELASCO, G.A.; PROAÑOS, J.; CORTES, W.; BALLESTEROS, I. 2011. Producción de azúcares fermentables por hidrólisis enzimática de cascarilla de arroz pretratada mediante explosión con vapor. Rev. Ion. 24:23-28.

22. SENE, L.; ARRUDA, P.V.; OLIVEIRA, S.M.M.; FELIPE, M.G.A. 2011. Evaluation of sorghum straw hemicellulosic hydrolysate for biotechnological production of xylitol by Candida guilliermondii. Braz. J. Microbiol. 42:1141-1146.

23. SHATALOV, A.A.; PEREIRA, H. 2012. Xylose production from giant reed (Arundo donax L.): Modeling and optimization of dilute acid hydrolysis. Carbohydr. Polym. 87:210-217.

24. SILVA, C.J.S.M.; ROBERTO, I.C. 2001a. Improvement of xylitol production by Candida guilliermondii FTI 20037 previously adapted to rice straw hemicellulosic hydrolysate. Lett. Appl. Microbiol. 32:248-252.
25. SILVA, C.J.S.; ROBERTO, I.C. 2001b. Optimization of xylitol production by Candida guilliermondii FTI 20037 using response surface methodology. Process Biochem. 36:1119-1124.

26. SILVA, C.J.S.M.; MUSSATTO, S.I.; ROBERTO, I.C. 2006. Study of xylitol production by Candida guilliermondii on a bench bioreactor. J. Food Eng. 75:115-119.

27. SOLEIMANI, M.; TABIL, L. 2014. Evaluation of biocomposite-based supports for immobilized-cell xylitol production compared with a free-cell system. Biochem. Eng. J. 82:166-173.

28. TOMOTANI, E.J.; ARRUDA, P.V.; VITOLO, M.; FELIPE, M.G.A. 2009. Obtaining partial purified xylose reductase from Candida guilliermondii. Braz. J. Microbiol. 40:631-635.

29. VILLALBA, M.; VÉlEZ, T.; ARIAS, M.; ARRÁZOLA, G. 2009. Producción de xilitol a partir de cascarilla de arroz utilizando Candida guilliermondii. Rev. Fac. Nal. Agr. 62(1):4897-4905.

30. ZHANG, W.; GENG, Y.A. 2012. Improved ethanol production by a xylose-fermenting recombinant yeast strain constructed through a modified genome shuffling method. Biotechnol. Biofuels. 5(1):46. DOI: 10.1186/1754-6834-5-46

31. ZOU, Y.; QI, K.; CHEN, X.; MIAO, X.; ZHONG, J. 2010. Favorable effect of very low initial kıa value on xylitol production from xylose by a self-isolated strain of Pichia guilliermondii. J. Biosci. Bioeng. 109:149152.

Recibido: Agosto 21 de 2016

Aceptado: Noviembre 29 de 2016

Cómo citar:

Manjarres-Pinzón, K.; Arias-Zabala, M.; Molina-Ramírez, Y.A.; Betancur-Nieto, M.I.; Rodríguez-Sandoval, E. 2016. Producción de xilitol por Candida guilliermondii a partir de fermentación de residuos de palma de aceite. Rev. U.D.C.A Act. \& Div. Cient. 19(2): 403-409. 